\title{
Modélisation de l'autovaporisation dans le cadre d'un modèle à deux fluides
}

\author{
T. Maciaszek, J.C. Micaelli, D. Bestion \\ CEA, Centre d'études nucléaires de Grenoble
}

Pour les besoins de l'analyse de sûreté des réacteurs à eau pressurisée (REP), un code de thermohydraulique diphasique eau-vapeur nommé CATHARE a été élaboré. Il s'agit d'un modèle monodimensionnel à deux fluides comportant 6 équations, c'est-à-dire un bilan de masse, d'énergie et d'impulsion pour chaque phase.

Le calcul des débits critiques à travers une brèche nécessite la modélisation de l'autovaporisation. Le flux de chaleur du liquide vers l'interface qui gouverne le phénomène est déterminé expérimentalement sur la base de mesures effectuées dans diverses tuyères. On présente tout d'abord une corrélation essentiellement empirique. Puis, l'analyse des mécanismes mis en jeu montrant la complexité du problème (nucléation, effets de la turbulence, effets bidimensionnels), une discussion sur des modèles à caractère plus physique est engagée.

Le code cathare et les besoins de l'analyse de sûreté des réacteurs à eau pressurisée

Les circuits primaires et secondaires d'un REP sont constitués d'un réseau de conduites de formes et de tailles variées. Des codes de thermohydrauliques de sûreté sont utilisés pour calculer des situations accidentelles, liées notamment à l'apparition d'une brèche; ces conduites sont alors le siège d'écoulements diphasiques eau-vapeur d'une grande variété. Les diamètres hydrauliques sont compris entre 1 et 80 centimètres, et la pression peut varier de 1 à 155 bars. La nécessaire prise en compte des déséquilibres mécanique et thermi- que des deux phases a conduit dans la dernière génération de codes à écrire des modèles à deux fluides comportant des bilans de masse, impulsion et énergie pour chacune des phases. Dans la phase actuelle de son développement, le code CATHARE, élaboré conjointement par le CEA, EDF, et Framatome, se limite à des calculs monodimensionnels. Une version future fera des calculs bidimensionnels pour traiter certains éléments du réacteur.

Dans les calculs monodimensionnels, les variables principales du système de 6 équations sont les valeurs moyennées sur la section de passage de la pression $\mathrm{P}, \mathrm{du}$ taux de vide $\alpha$, des vitesses vapeur $V_{V}$ et liquide $V_{L}$, et des enthalpies $h_{V}$ et $h_{L}$.

\section{Modeling flashing flows in a two fluid code.}

CATHARE is a 2-phase thermohydraulic code elaborated for the Pressurized Water Reactor safety analysis. It is a 1-Dimension 2-Fluid 6-Equation model with a mass, momentum and energy balance for each phase.

The calculation of the critical mass flow rate through a break in the primary circuit requires the modelization of the flashing process. The liquid-interface heat flux which governs the phenomenon is experimentally determined in various test nozzles. First an essentially empirical correlation is presented. Then a survey of the physical mechanisms involved in the flashing process demonstrate the complexity of the problem (nucleation delay, turbulence effects, 2-dimensionnal effects,...). Modelizations based on physical investigations are also discussed. 


$$
\begin{aligned}
& \frac{\partial}{\partial t} A \alpha \rho_{V}+\frac{\partial}{\partial Z} A \alpha \rho_{V} V_{V}=A \Gamma \\
& \frac{\partial}{\partial t}(1-\alpha) \rho_{\ell}+\frac{\partial}{\partial Z} A(1-\alpha) \rho_{L} V_{L}=-A \Gamma \\
& \frac{\partial}{\partial t} A \alpha \rho_{V} V_{V}+\frac{\partial}{\partial Z} A \alpha\left(\rho_{V} V_{V}^{2}+P\right) \\
& =-A \tau_{P V}+A I+A \alpha \rho_{V} g \\
& \frac{\partial}{\partial t} A(1-\alpha) \rho_{L} V_{L}+\frac{\partial}{\partial Z}(1-\alpha) A\left(\rho_{\ell} V_{L}^{2}+P\right) \\
& =-A \tau_{P \ell}-A I+A(1-\alpha) \rho_{L} g \\
& \frac{\partial}{\partial t} A \alpha\left[\rho_{V}\left(h_{V}+\frac{V_{V}^{2}}{2}\right)-P\right]+\frac{\partial}{\partial Z} A \alpha \rho_{V} V_{V}\left(h_{V}+\frac{V_{V}^{2}}{2}\right) \\
& =A Q_{V}+A \alpha_{V} V_{V} g+A Q_{P V} \\
& \frac{\partial}{\partial t}(1-\alpha)\left[\rho_{L}\left(h_{L}+\frac{V_{L}^{2}}{2}\right)-P\right] \\
& +\frac{\partial}{\partial Z} A(1-\alpha) \rho_{L} V_{L}\left(h_{L}+\frac{V_{L}^{2}}{2}\right)= \\
& A Q_{L}+A(1-\alpha) \rho_{L} V_{L} g+A Q_{P L}
\end{aligned}
$$

On trouve dans les seconds membres des termes représentant les transferts à la paroi d'impulsion $\tau_{P K}$ et d'énergie $Q_{P K}$, ainsi que des termes de transfert interfaciaux de masse, d'impulsion et d'énergie:

$$
\begin{gathered}
\Gamma=-\frac{q_{\ell e}+q_{V e}}{h_{V}-h_{L}} \\
I=\tau_{i}+\Gamma V_{i}-M_{i} \\
Q_{K}=q_{K e}+\Gamma\left(h_{K}+\frac{V_{i}^{2}}{2}\right) \pm M_{i} \cdot V_{i}
\end{gathered}
$$

\section{Méthode générale de développement}

Pour que le système d'équations soit fermé, tous les termes de transfert doivent être modélisés en fonction des 6 variables principales. A cette fin, une série d'expériences analytiques dites "à effets séparés " a été réalisée, chacune permettant d'étudier un phénomène physique particulier. Le dépouillement et l'analyse des données d'expérience conduit à l'élaboration de corrélations ou de "lois constitutives " pour les divers transferts concernés. Ensuite le code pourvu de son jeu complet de corrélations effectue des calculs de qualification sur un vaste programme expérimental afin de vérifier la cohérence globale des modèles.

Pour l'autovaporisation, un modèle semi-empirique, c'est-à-dire comportant un minimum d'hypothèses physiques, est établi en cherchant le meilleur compromis entre la précision et la généralité. Parallèlement, des études analytiques plus fines servent à mieux mesurer les limites du modèle et à guider les futurs développements.
Modélisation des débits critiques et de l'autovaporisation

\section{Support expérimental - Méthode de dépouil- lement}

Plusieurs expériences de débits critiques en tuyère ont été utilisées pour l'élaboration et la vérification des modèles. La chute brutale de pression subie par le fluide provoque une forte vaporisation contrôlée par le flux de chaleur du liquide vers l'interface qui correspond au terme qle des équations du modèle. Des études de sensibilité montrent que pour des conditions sous saturées en amont, la précision requise sur le terme qle peut être très grande pour une prédiction correcte des débits. Par contre, pour des conditions génératrices saturées diphasiques, le calcul des débits est beaucoup plus sensible au modèle mécanique, c'est-à-dire aux frottements pariétaux et interfaciaux, qu'aux transferts de chaleur. On présente ici plus particulièrement les situations gouvernées par la vaporisation.

La boucle Super Moby-Dick a testé dans une gamme de pressions de 10 à 120 bars plusieurs tuyères présentées ci-dessous (fig. 1). Par ailleurs, l'expérience MARVIKEN a étudié en transitoire des tuyères de grand diamètre.

Les tuyères SMD 1 et 2 ont un convergent spécialement profilé pour minimiser les effets bidimensionnels liés aux gradients radiaux de pression. La tuyère $3 a$, au contraire, un convergent plus brutal en arc de cercle qui peut provoquer des décollements. C'est initialement la tuyère 1 qui, comportant des mesures fines de la pression et du taux de vide, a fait l'objet d'un dépouillement systématique. La répartition radiale du taux de vide était également obtenue par une méthode de densitométrie à rayons X multi-faisceaux. La vapeur apparaissait à la paroi ou près de la paroi, puis gagnait toute la section de passage.

Le dépouillement s'effectue à l'aide d'un code inverse : il s'agit de résoudre le système d'équations de CATHARE en remplaçant l'inconnue pression $\mathrm{P}$ (connue par la mesure), par l'inconnue qle. On obtient ainsi une banque de données $\left[P, H_{L}, H_{V}, \alpha, V_{L}, V_{V}, q l e\right]$. Il reste à corréler le terme qle.

\section{Modèle de qle semi-empirique}

Un premier modèle à caractère essentiellement empirique a ainsi été cherché sous la forme:

$$
q l e=A\left(P, H_{L}, H_{V}, \alpha, V_{L}, V_{V}, D_{H}\right)\left(T_{L}-T_{\text {sat }}\right)
$$

Il s'agit d'un modèle algébrique fonction uniquement des variables locales et non de leurs dérivées. Il exclut toute prise en compte d'un effet d'histoire tel que l'état de développement d'une couche limite ou de l'influence de la forme du convergent sur un éventuel décollement, etc. Il est apparu que le coefficient $A$ croissait fortement avec $V_{L}$ mais ne dépendait pas notablement de la pression. De plus, la comparaison des essais SUPER MOBY-DICK et MARVIKEN n'a pas révélé une forte dépendance en diamètre et l'examen de l'ensemble des essais a conduit à choisir une forte croissance avec le taux de vide. Ajoutant à cela des considérations dimensionnelles, on a obtenu : 


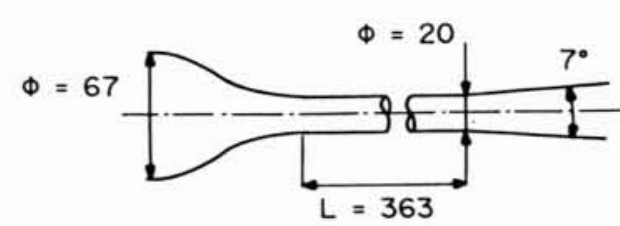

SUPER MOBY-DICK I

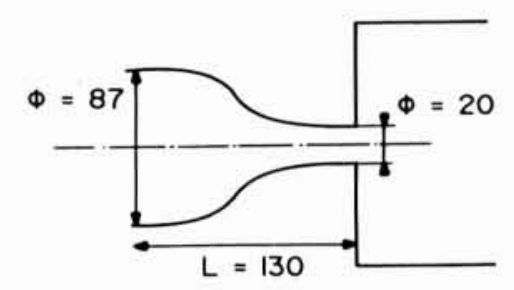

SUPER MOBY-DICK 2

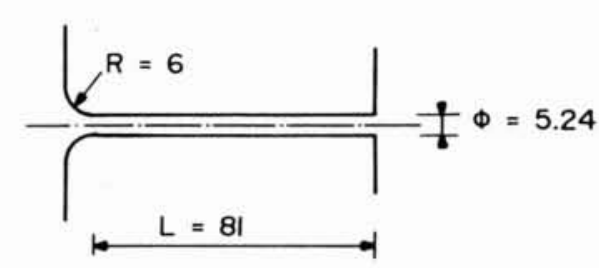

SUPER MOBY-DICK 3

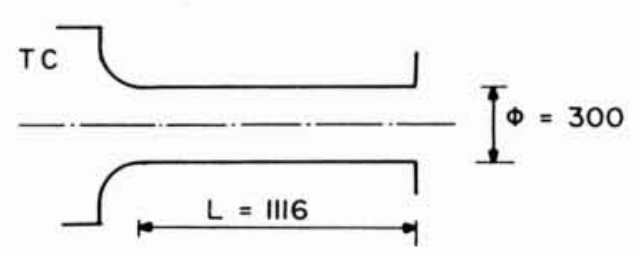

MARVIKEN (TEST 17)

1. Géométries des tuyères testées (dimensions en $\mathrm{mm}$ )

$q l e=-f(\alpha) \times \frac{\rho_{L}^{2} k_{\ell}}{\mu_{L}^{2} C p_{L}} \times V_{L}^{2}\left(T_{L}-T_{\mathrm{sat}}\right)$

$f(\alpha)=1.210^{8} e^{4.5 \alpha}$

Un modèle de seuil de nucléation est également nécessaire pour faire démarrer l'évaporation à partir d'une certaine surchauffe de liquide. La réf. (1), reprenant des travaux antérieurs, propose le seuil suivant:

$$
P_{\text {début d'ébullition }}=0.98 P_{\text {sat }}\left(T_{L}\right)
$$

Ce modèle, appliqué au calcul des diverses tuyères, donne les résultats présentés sur les figures $2,3,4$ et 5 (page suiv.). On note une bonne qualité d'ensemble, excepté pour les très faibles sous saturations où l'écart peut atteindre $20 \%$ avec l'expérience. On a, avec cette corrélation, cherché une précision homogène sur l'ensemble des expériences.

\section{Analyse critique des modèles d'autovapori-} sation

\section{Test d'un modèle de convection}

De l'analyse, due à Berne (réf. 2), des mécanismes physiques liés à l'autovaporisation, on a retenu deux points essentiels :

- La taille des bulles, et donc la surface d'échange, est principalement contrôlée par la turbulence.

- La convection sur les bulles semble être le mécanisme de transfert de chaleur prépondérant.

On teste donc avec CATHARE un modèle issu de cette analyse. En reprenant la relation suivante entre nombres de Nusselt et de Peclet, $\mathrm{Nu}=(2 / \sqrt{\pi}) \mathrm{Pe}^{1 / 2}$, on obtient l'expression du qle:

$$
\text { qle }=\frac{6}{\sqrt{2 \pi}} \alpha\left[\frac{k_{L} \rho_{L} C p_{l}\left(V_{V}-V_{L}\right)}{r^{3}}\right]^{1 / 2}\left(T_{L}-T_{\text {sat }}\right)
$$

On considère qu'il n'existe qu'un seul rayon de bulle $r$ par section d'écoulement. Les bulles croissent d'abord à nombre constant (on a alors $r=[3 \alpha / 4 \pi n]^{1 / 3}$ et on choisit $n=10^{10} \mathrm{~m}^{-3}$ ) jusqu'à la taille critique de fractionnement où le rayon est calculé en prenant un nombre de Weber de 1.2. Le fractionnement est fait par les structures turbulentes de même taille que la bulle. L'échelle de vitesse est donnée par la relation $v^{\prime}=2(d \varepsilon)^{2 / 3}$ valable dans la zone inertielle du spectre de turbulence. Le taux de dissipation est ensuite estimé à l'aide de la vitesse de frottement $V^{\star}$ et on obtient:

$$
r_{\text {fractionnement }}=\frac{1}{2} W e^{3 / 5}\left(\frac{\sigma}{\rho_{L}}\right)^{3 / 5} \frac{5}{7}\left(\frac{K D}{2 V^{\star 3}}\right)^{2 / 5}
$$

Le modèle de frottement interfacial correspond à un coefficient de traînée sur une bulle égal à 1 .

La confrontation de ce modèle aux données de Super Moby Dick -1- conduit à appliquer un facteur correctif fonction du taux de vide au qle: $f(\alpha)=(1-\sqrt{\alpha})^{1.8}$.

Les calculs effectués avec ce modèle sont présentés sur les figures 2 à 5 . L'erreur maximale a été réduite sur les essais SMD 1 et 2, mais les débits sont notablement surestimés sur SMD 3 et Marviken.

\section{Discussion}

En l'état actuel des études sur les modèles d'autovaporisation, on établit le bilan suivant :

- Dans la recherche d'une grande généralité, l'utilisation d'un modèle à fort degré d'empirisme a permis d'obtenir une qualité d'ensemble en progrès par rapport aux modèles homogènes.

- L'analyse physique a pu discerner des mécanismes essentiels contrôlant l'autovaporisation. Leur modélisation rencontre des difficultés car de nombreuses hypothèses doivent être faites sur des paramètres encore inaccessibles à la mesure. Aussi le modèle physique testé a nécessité un réajustement sur l'expérience, et, par ailleurs, il a perdu en généralité ce qu'il a gagné en précision. 


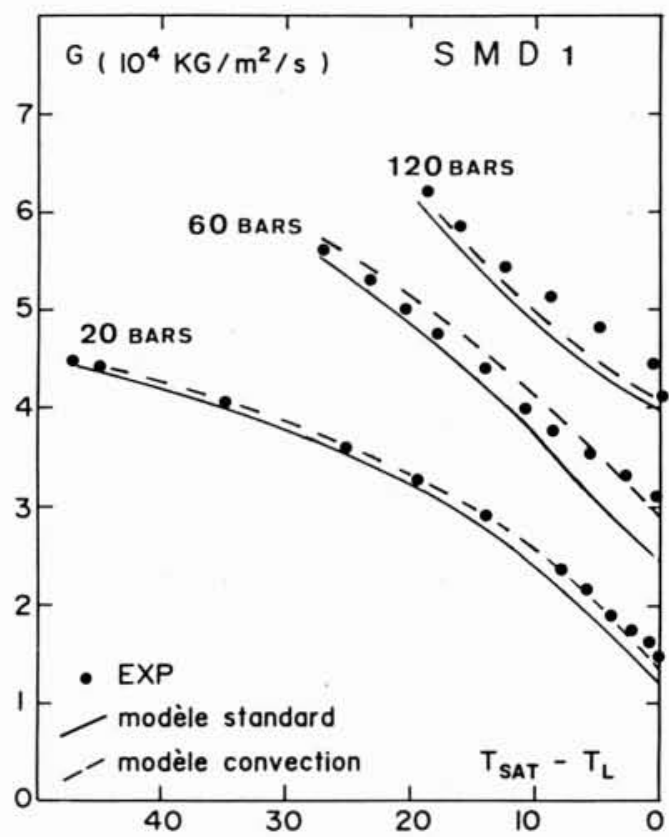

2. Tuyère Super Moby-Dick -1- Débit critique en fonction de la sous-saturation

Comparaison expérience - modèle standard de Cathare modèle de convection

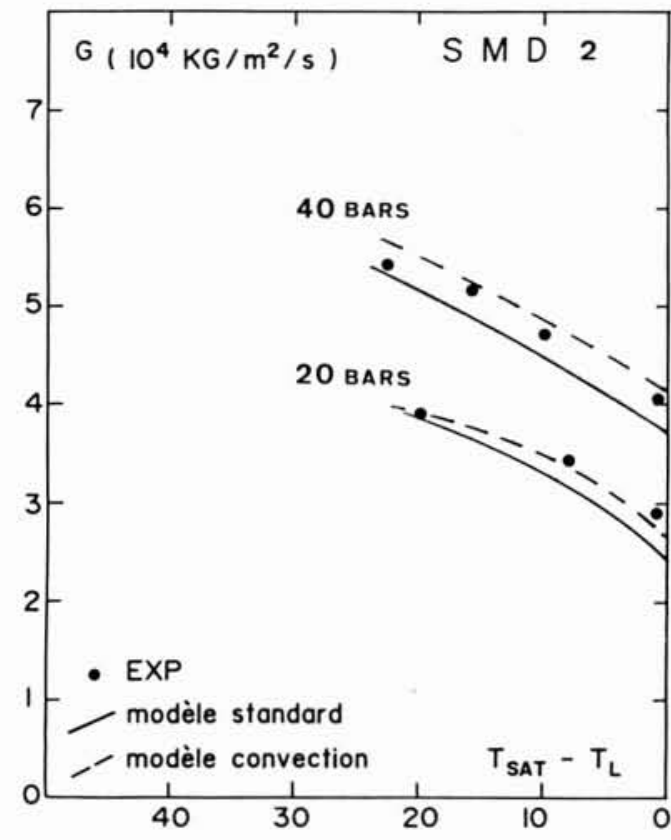

3. Tuyère Super Moby-Dick -2

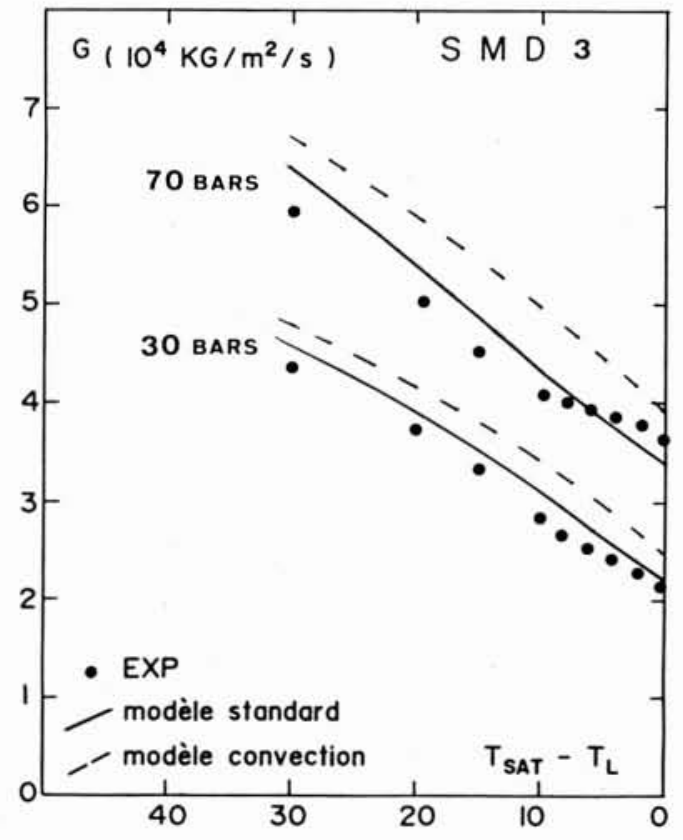

4. Tuyère Super Moby-Dick -3-

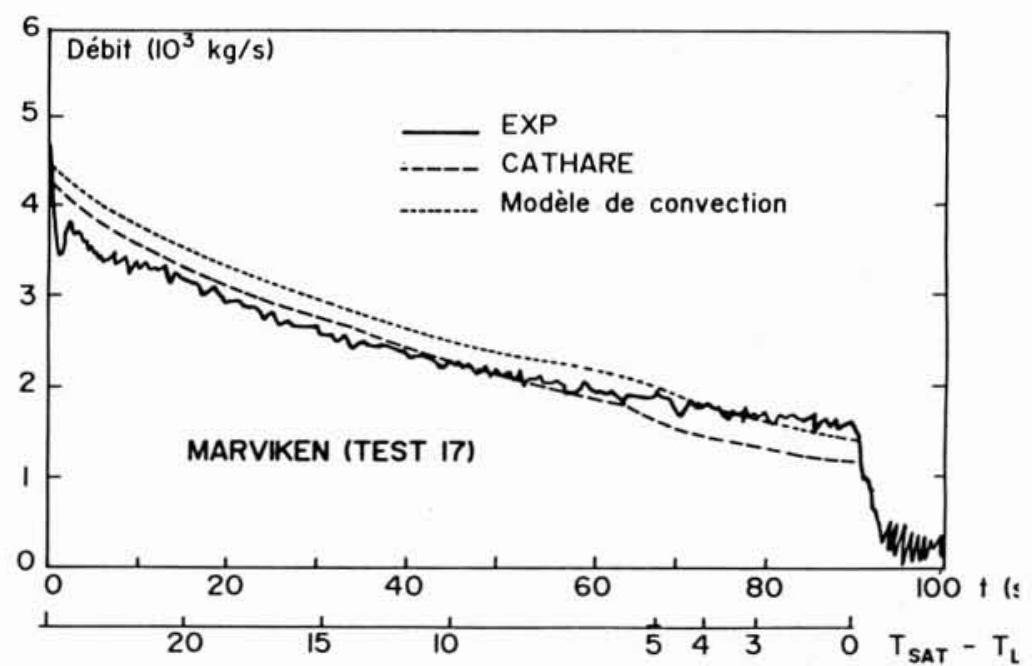

5. Essai Marviken 17. Débit critique en fonction de la sous. saturation (ou du temps)

Comparaison expérience - modèle standard de Cathare modèle de convection. 
Ainsi divers points viennent dresser les limites du modèle à 6 équations :

- Si les caractéristiques turbulentes contrôlent bien la surface d'échange, on peut difficilement se passer de décrire leur évolution à la traversée d'un convergent. Par ailleurs, l'influence des bulles sur la turbulence est très méconnue.

- La validité d'un modèle monodimensionnel n'est plus garantie hors du domaine des conduites longues et de section constante. A cet égard, on peut noter que les essais les moins bien calculés par le modèle de convection sont ceux qui ont des convergents brutaux (SMD 3 et Marviken).

En définitive, des progrès limités peuvent être attendus dans le cadre du modèle à 6 équations. A plus long terme, on peut envisager de rajouter des équations de transport ou de passer à une description bidimensionnelle.

\section{Notations}

$\begin{array}{llll}A & : \text { section de passage } & N u & : \text { nombre de Nusselt } \\ C p l & : \text { chaleur massique à pression constante du } & P e & : \text { nombre de Peclet } \\ & \text { liquide } & P & : \text { pression } \\ d & : \text { échelle de longueur (= diamètre de bulle) } & T l & : \text { température du liquide } \\ D \cdot D h & : \text { diamètre de la conduite }- \text { diamètre } & T_{\text {sat }} & : \text { température de saturation } \\ & \text { hydraulique } & V k & : \text { vitesse de la phase } \mathrm{k} \\ g & : \text { accélération de la pesanteur } & \mu_{1} & : \text { viscosité du liquide } \\ h_{k} & : \text { enthalpie de la phase k } & \Gamma & : \text { taux de vaporisation }(\mathrm{kg} / \mathrm{m} / \mathrm{s}) \\ k l & : \text { conductivité thermique du liquide } & \rho_{k} & : \text { densité de la phase } k \\ K & : \text { constante de Karman } & \varepsilon & : \text { taux de dissipation } \\ M i & : \text { terme de masse ajoutée } & \sigma & : \text { tension superficielle }\end{array}$

\section{Références}

[1] Sekri Ahcène. - Cinétique d'ébullition de l'eau en décompression rapide.

Thèse de Docteur Ingénieur, Univ. Louis Pasteur de Strasbourg 1982.
[2] Berne Philippe. - Contribution à la modélisation du taux de production de vapeur par autovaporisation dans les écoulements diphasiques en conduite.

Thèse présentée à l'ECP, 1983.

\section{Discussion}

Président : M. R. SÉMÉRIA

Le Président. - Je remercie M. BESTION de son exposé. Y a-t-il des questions à son sujet ?

M. DELHAYE. - Quelle est l'influence du nombre de bulles par unité de volume sur les résultats obtenus avec le modèle de convection?

M. BESTION. - Dans les calculs présentés, on atteignait très vite le rayon de fractionnement. On a donc trouvé une faible sensibilité au nombre de bulles initial.

M. Delhaye. - Ce taux de nucléation a fait l'objet de travaux récents au Rensselaer Polytechnic Institute. Le Pr. O.C. JONES Jr a publié deux articles sur ce sujet.

Le Président. - Nous allons passer à la deuxième présen. tation de communication. Celle-ci a été rédigée par plusieurs personnes appartenant à deux organismes.

La première partie sera présentée par M. FRANC, de l'Institut de mécanique de Grenoble, suivie d'une présentation complé. mentaire de M. MÉnoret, de la Société ALSTHOM A.C.B. CERG, ex NEYRTEC. Cette communication concerne la cavitation, vaste domaine.

\section{Adresse des auteurs \\ Messieurs T. Maciaszek
J.C. Micaelli \\ D. Bestion \\ CEA-CENG \\ $85 \mathrm{X}$ \\ 38041 Grenoble Cedex \\ Tél. : 76.88 .44 .00}

